\title{
Stereotactic body radiation therapy for primary liver tumors with adverse factors
}

\author{
Takuya Shimizuguchi'1, Jun Imamura², Simpei Hashimoto', Katsuyuki Karasawa1 \\ 'Department of Radiation Oncology, Cancer and Infectious Diseases Center Komagome Hospital, Tokyo 113-8677, Japan. \\ ${ }^{2}$ Department of Hepatology, Cancer and Infectious Diseases Center Komagome Hospital, Tokyo 113-8677, Japan.
}

Correspondence to: Dr. Takuya Shimizuguchi, Department of Radiation Oncology, Cancer and Infectious Diseases Center Komagome Hospital, 3-18-22 Hon-komagome, Bunkyo-ku, Tokyo 113-8677, Japan. E-mail: shimizuguchi@cick.jp

How to cite this article: Shimizuguchi T, Imamura J, Hashimoto S, Karasawa K. Stereotactic body radiation therapy for primary liver tumors with adverse factors. Hepatoma Res 2020;6:66. http://dx.doi.org/10.20517/2394-5079.2020.51

Received: 14 May 2020 First Decision: 22 Jun 2020 Revised: 13 Aug 2020 Accepted: 18 Aug 2020 Published: 12 Oct 2020

Academic Editors: Su Pin Choo, Guang-Wen Cao, David Tan Boon Harn Copy Editor: Cai-Hong Wang Production Editor: Jing Yu

\begin{abstract}
Aim: To test the efficacy and safety of liver stereotactic body radiation therapy (SBRT) in patients who harbor adverse factors.

Methods: We retrospectively evaluated the outcomes of liver SBRT in a single cancer center. We invented criteria consisting of two physical factors and two tumor factors to measure the treatment difficulty in each case. The clinical outcomes and toxicity were evaluated by stratification of the harboring factors.
\end{abstract}

Results: A total of 24 (23 hepatocellular carcinoma and 1 intrahepatic cholangiocarcinoma) patients were eligible for this study, with a median follow-up duration of 18 months. Of all eligible patients, 21 patients ( $88 \%$ ) had one or more factors. The local control, progression-free survival, and overall survival rates for all patients at 2 years were $89 \%, 42 \%$, and $76 \%$ respectively. In the patients with physical and tumor adverse factors, local control/progression-free survival/overall survival rates at 2 years were $100 \% / 42 \% / 69 \%$ and $80 \% / 23 \% / 78 \%$, respectively. The subgroup of 11 patients with 2 or more factors showed comparable local control rate at 2 years to the subgroup of 13 patients with 0 to 1 factors $(100 \% v s .86 \%, P=0.59)$. One patient (4.2\%) experienced a decline in the Child-Pugh score by 2 points at 3 months after the treatment. Grade 2 to 3 gastrointestinal toxicity was observed in three patients.

Conclusion: SBRT showed a high local control rate with acceptable toxicity for the group of liver cancer patients harboring both physical and tumor adverse factors as long as conducted following patient selection and dose constraints that were used in this study.

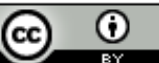

(C) The Author(s) 2020. Open Access This article is licensed under a Creative Commons Attribution 4.0 International License (https://creativecommons.org/licenses/by/4.0/), which permits unrestricted use, sharing, adaptation, distribution and reproduction in any medium or format, for any purpose, even commercially, as long as you give appropriate credit to the original author(s) and the source, provide a link to the Creative Commons license, and indicate if changes were made. 
Keywords: Hepatocellular carcinoma, stereotactic body radiation therapy, vulnerable patients

\section{INTRODUCTION}

Hepatocellular carcinoma (HCC) is one of the most common malignant diseases in the world and the fourth most common cause of cancer death in $\operatorname{Japan}^{[1,2]}$. Treatment of HCC consists of surgical resection, radiofrequency ablation, transarterial chemoembolization, and use of systemic anticancer agents ${ }^{[3,4]}$. Moreover, the use of stereotactic body radiation therapy (SBRT), a nonsurgical local treatment, has rapidly increased in the past decade owing to its ability to deliver a precise radiation dose with modern radiation oncology devices and techniques ${ }^{[5]}$. Referring to the eligibility criteria for prospective liver SBRT trials, the ideal candidate for the treatment is considered to: (1) be in fair general condition; (2) have adequate liver function [Child-Pugh (CP) score 5 to 6]; and (3) a tumor size, number, and location that are amenable to dose constraints for organs at risk (OAR) in treatment planning ${ }^{[6-8]}$. However, patients with liver tumors who are referred to SBRT are usually unsuitable for surgery or other local therapies due to comorbidity and impaired liver function. In addition, in daily practice, a considerable proportion of tumors are unfit for typical SBRT planning due to the radiation therapy planning dose constraint. Both physical factors and tumor factors affect the decision of treatment choice. The more patient factors that do not meet clinical trial criteria, the higher tendency to select non-localized treatment or conservative options instead of liver SBRT in clinical practice. To the best of our knowledge, no study has directly tested the impact of those composite adverse factors in liver SBRT. Therefore, this study aimed to evaluate the efficacy and feasibility of liver SBRT for cases with originally defined adverse factors.

\section{METHODS}

\section{Patient data}

We planned a retrospective study of patients with liver tumors who were treated by liver SBRT at the Tokyo Metropolitan Cancer and Infectious Diseases Center, Komagome Hospital between 2014 and 2019. The institutional patient database was used to screen patients. The eligibility criteria were as follows: (1) diagnosis of primary liver malignancy; (2) first liver SBRT (subsequent SBRT episodes were excluded); and (3) definitive treatment (not palliative intent). Patient characteristics, laboratory testing, imaging findings, and SBRT parameters were extracted from the medical records, and the comorbidities of the patients were evaluated by the Charlson comorbidity index ${ }^{[9]}$. This study was approved by the Institutional Review Board of Tokyo Metropolitan Cancer and Infectious Diseases Center, Komagome Hospital (\#2450).

\section{Treatment procedure}

To visualize the liver tumor position, a fiducial marker (Visicoil, RadioMed LLC, California, USA; length: $10 \mathrm{~mm}$, diameter: $0.75 \mathrm{~mm}$ ) was implanted next to the target by the hand of a hepatologist 2 weeks prior the treatment. Subsequently, a 4-dimensional computed tomography (CT) image with contrastenhancement was obtained for treatment planning. Treatments were conducted using a Vero 4DRT system (Mitsubishi Heavy Industry, Tokyo, Japan), which equips the tracking system.

Gross tumor volume was defined in the planning CT scan with the guide of contrast-enhanced magnetic resonance imaging image fusion. The clinical target volume (CTV) was obtained by adding a 4-mm margin to the gross tumor volume within the liver volume, while the planning target volume (PTV) was generated by adding a margin to the CTV for uncertainness of setup and tracking (usually a 5-6 mm margin to the CTV).

A total of 40 Gy in 5 fractions over two weeks was prescribed to the $70 \%$ isodose fitting to the PTV. The modified prescription was allowed to achieve dose constraints: A reduced prescription dose (down to 32 Gy) 
Table 1. Criteria of adverse factors

\begin{tabular}{ll}
\hline Factor & Criteria \\
\hline $\begin{array}{l}\text { 1. Physical factor } \\
\text { General condition } \\
\text { Liver function }\end{array}$ & ECOG performance status $=2$ or above or Charlson comorbidity index $=5$ or above \\
$\begin{array}{l}\text { 2. Tumor factor } \\
\text { Tumor persistence }\end{array}$ & Three or more previous liver tumor treatments $=7$ or above or normal liver volume $<1000 \mathrm{~mL}^{\star}$ \\
Planning difficulty & Target is in immediate contact with the gastrointestinal tract or tumor size $>5 \mathrm{~cm}$ \\
\hline
\end{tabular}

*Liver volume subtracted by the gross target volume; **either surgery, radiofrequency ablation, or transarterial chemoembolization for liver tumor. CP score: Child-Pugh score; ECOG: European Clinical Oncology Group

in 5 fractions was selected to reduce the normal liver dose, while a more fractionated schedule (40 Gy in 10 fractions) was selected for cases in which the PTV was in direct contacted with the OARs. Dose constraint criteria for the liver were volumes receiving $20 \mathrm{~Gy}(\mathrm{~V} 20)<20 \%$. Dose for digestive tube was restricted below $30 \mathrm{~Gy}$ in 5 fractions to at most $0.5 \mathrm{cc}$ of the volume.

\section{Evaluation of outcomes and statistical considerations}

To measure the difficulty of the treatment in each case, we invented criteria consisting of two physical factors and two tumor factors as shown in Table 1. Each adverse factor was weighted equally, and the patients were categorized by the number of harboring factors.

Patients were usually evaluated by liver function blood test and CT or magnetic resonance imaging every 3 to 6 months after the treatment. Endoscopy was not routinely performed unless the patient had gastrointestinal symptoms. Local control was defined as freedom from radiological progression ( $>20 \%$ growth in the diameter), and data were censored when patients were lost to follow-up or died without local progression. Progression-free survival was defined as the duration to any liver tumor recurrence and death. Overall survival (OS) was defined as the time until to death from any cause. All the indicators were counted from the initial day of SBRT, and rates were estimated by the Kaplan-Meier method. Patients who had two or more adverse factors were compared to those who did not, in terms of local control rate. Toxicity was evaluated using the Common Terminology Criteria for Adverse Events version 4.0. Liver function before and after SBRT was evaluated with the CP scoring system.

\section{RESULTS}

A total of 24 patients were eligible for this study between January 2014 and December 2019. The median follow-up duration was 18 months, and the patient characteristics are listed in Table 2. All the patients were pathologically or radiologically diagnosed with primary liver malignancy; one patient was diagnosed with intrahepatic cholangiocarcinoma, the remainder of patients were diagnosed with HCC. A modified SBRT prescription was used in 9 patients (an OAR dose constraint in 3 patients and a liver dose constraint in 6 patients). Of all eligible patients, 21 patients (88\%) had one or more adverse factors, 16 patients (67\%) had physical factors, 13 patients (54\%) had tumor factors, and 11 patients (46\%) had two or more adverse factors. The number of patients who met the criteria of the adverse factor was 10 for general condition, 10 for liver function, 9 for tumor persistence, and 7 for planning difficulty.

\section{Outcomes}

The local control, progression-free survival, and OS rates for all patients at 2 years were $89 \%, 42 \%$, and $76 \%$, respectively [Figure 1]. One patient (patient number 8 in Table 2) experienced local progression within the PTV 20 months after SBRT was confirmed on CT. In the patients with physical and tumor adverse factors, local control/progression-free survival/OS rates at 2 years were $100 \% / 42 \% / 69 \%$ and $80 \% / 23 \% / 78 \%$, respectively. The subgroup of 11 patients with 2 or more (13 patients with 0 to 1 ) adverse factors showed 
Table 2. Patient baseline characteristics

\begin{tabular}{ll}
\hline Characteristic & Value \\
\hline Age, median (range), years & $72(57-93)$ \\
sex $\quad$ Male & 19 \\
Female & 5 \\
ECOG performance status & 5 \\
0 & 16 \\
1 & 3 \\
2 & \\
Charlson comorbidity index ${ }^{[9]}$ & 15 \\
3 to 4 & 9 \\
5 or more & \\
Underlying liver disease & 7 \\
HCV infection & 4 \\
HBV infection & 4 \\
Alcohol & 5 \\
Non-alcoholic steatohepatitis & 4 \\
Other/none & \\
O & 9 \\
1 to 2 & 6 \\
3 or more & 9 \\
Previous treatment course & \\
ECOG: European Oncology Study Group; HVB: hepatitis B virus; & HCV: hepatitis C virus
\end{tabular}

a comparable local control rate at 2 years $100 \%(86 \%)(P=0.59)$ [Figure 2]. In the 11 patients who experienced non-local progression, 9 had intrahepatic recurrences as the first site of recurrence, one had both intrahepatic and regional lymph node recurrence, and one had lung metastasis.

\section{Toxicity}

One patient (4.2\%) experienced a decline in CP score by 2 points 3 months after the treatment, although it remains unclear whether SBRT directly worsened the liver function since HCC developed rapidly after the treatment. Grade 2 or greater gastrointestinal toxicity was observed in three patients: One patient experienced Grade 3 cholangitis 3 months after the SBRT for S4/8 tumor; another patient experienced Grade 2 esophagitis 2 months after SBRT for S8 tumor; and the other patient with an S5/6 tumor experienced Grade 2 lower gastrointestinal hemorrhage. The list of SBRT treatment planning parameters, liver function before and after treatment, and toxicity are shown in Table 3.

\section{DISCUSSION}

The current study evaluated the safety and efficacy of liver SBRT in patients with combined treatment difficulty. An equally high local control rate with acceptable toxicity was demonstrated in patients with both physical and tumor adverse factors.

The patient selection criteria of the prospective liver SBRT trials, and the patient characteristics that were actually included denote the group of patients in which the feasibility of SBRT has been confirmed. Existing prospective studies of liver SBRT mainly recruited patients with CP A diseases, or those with at least CP B score $7^{[6-8]}$. Additionally, most of the patients who participated in those prospective studies were categorized as ECOG PS 0 or 1. Thus, the safety and efficacy of liver SBRT in unfit patients has not been demonstrated in prospective trials. Although tumor location is not necessarily regarded as a crucial factor in liver SBRT eligibility, substantial attention is paid to dose constraints when the tumor is in contiguity with OAR. Furthermore, controversy exists in relation to the dose constraint for the digestive tube, although previous studies encourage clinicians to reduce the dose for the digestive tube below the prescription dose ${ }^{[10]}$. 

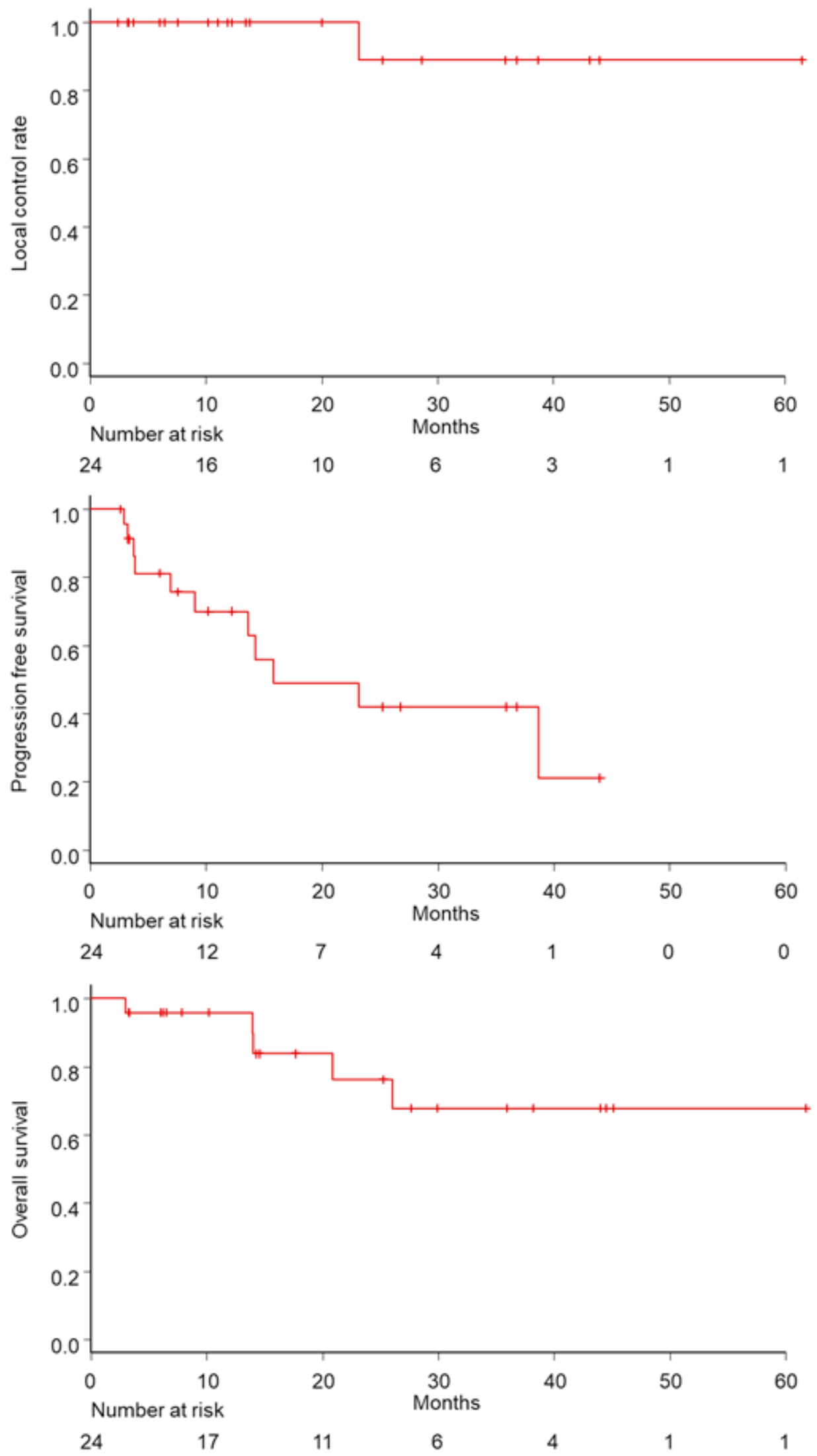

Figure 1. Local control rate (top), progression-free survival rate (middle), and overall survival rate (bottom) for all patients in this study 

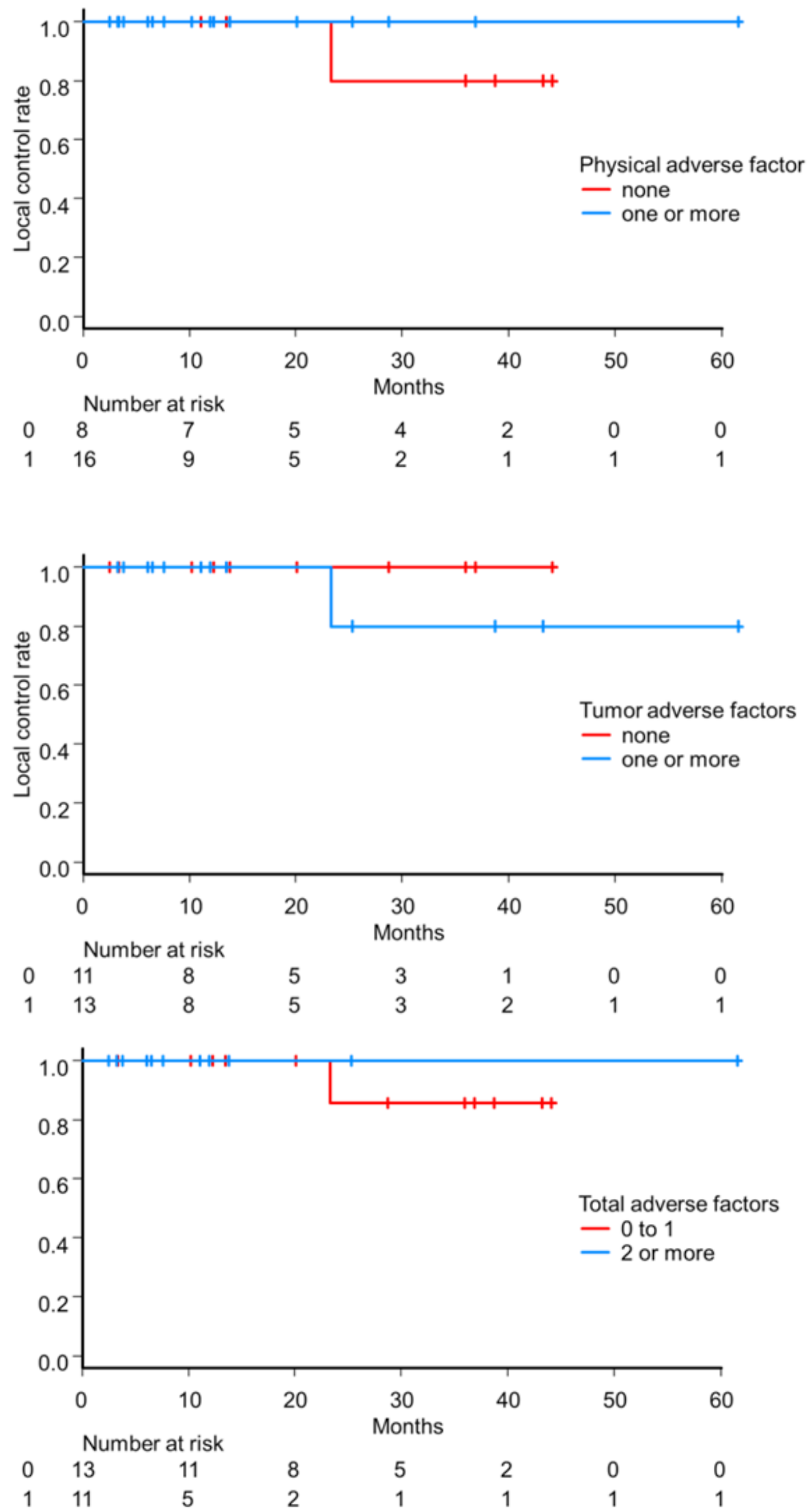

Figure 2. Local control rate for patients with or without physical adverse factor (top), for patients with or without (middle) and for patients with 2 or more total factors or not (bottom) 


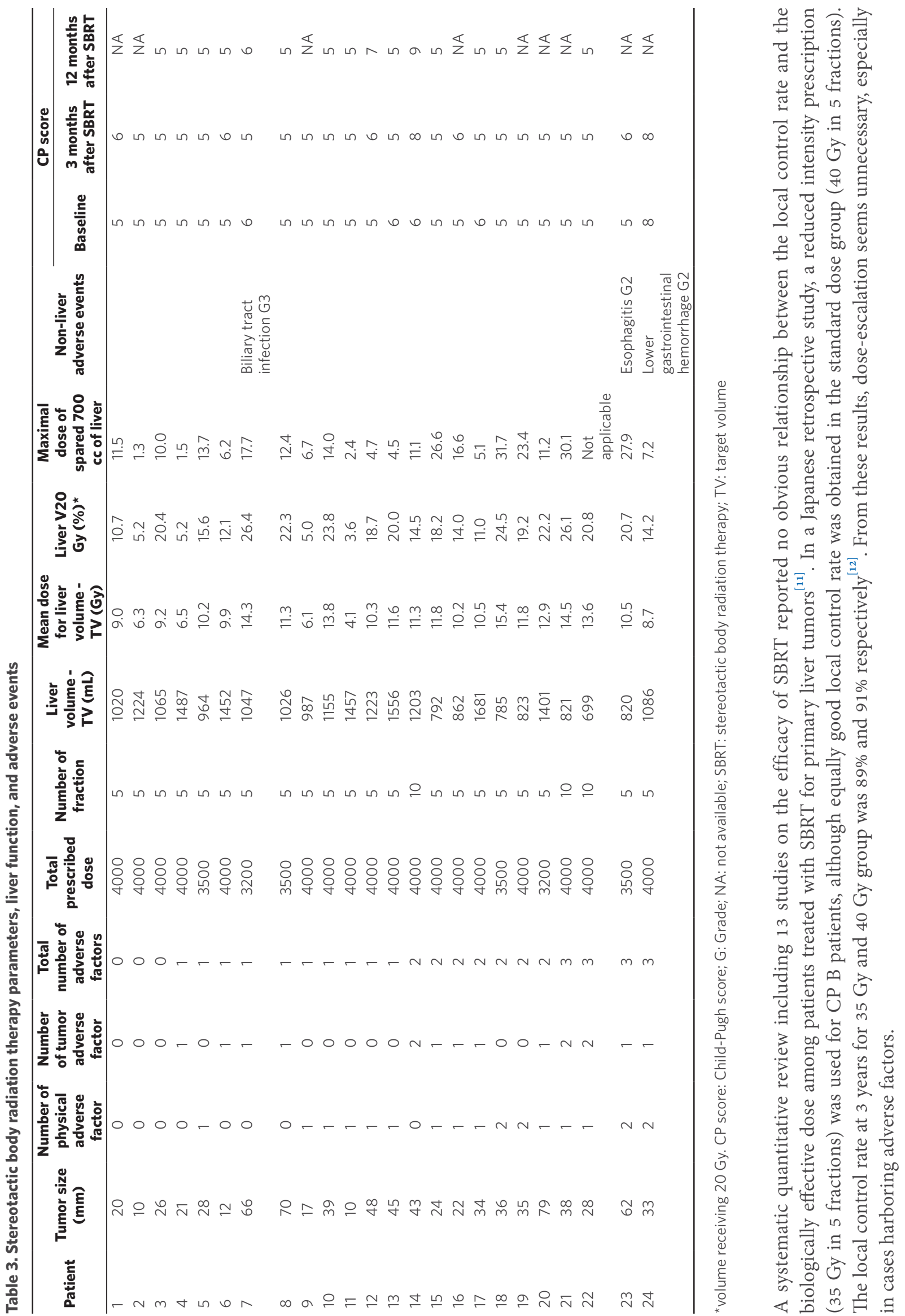


Studies involving patients with more advanced liver tumors, or in those with impaired liver function, reported a greater risk of treatment-related toxicity. In the largest prospective Phase I/II study in patients with multiple HCC, $68 \%$ of patients had two or more lesions, resulting in a mean liver dose $>20$ Gy in a fraction of patients. Seven patients experienced Grade 5 adverse events (liver failure for five patients). Additionally, $29 \%$ of the patients experienced deterioration in the CP class 3 months after SBRT ${ }^{[6]}$. A study involving patients with poor liver function (CP B or C) reported that the OS rate at 1 year was $32 \%$ and $63 \%$ of patients experienced a decline in CP score by 2 or more points at 3 months ${ }^{[13]}$. For challenging cases, the indication for patient-oriented SBRT should be decided based on HCC prognosis, liver function, patients request, and other options.

The originally defined adverse factors, both physical and tumor factors, in this study did not seem to be a crucial issue in the liver SBRT. Of the total included patients, $88 \%$ had at least one and $46 \%$ had two or more adverse factors, although a high local control rate and acceptable toxicity were achieved. Thus, our approach appears reasonable in terms of patient selection and toxicity management.

In the future, novel technologies might change the borderline of the indication of liver SBRT. Magnetic resonance imaging linac provides real-time high contrast image-guided radiation therapy, which enables highly accurate dose delivery with a minimal PTV margin ${ }^{[14]}$. Moreover, proton beam RT has an advantage on dose distribution over standard proton-based radiation in terms of its physical profile, and some prospective trials have reported the clinical outcome ${ }^{[15]}$. However, as there is no direct comparison to date, its clinical advantage over photon-SBRT remains unclear. To clarify this point, a Phase III randomized trial NRG-GI003 (NCT03186898) is open for accruing patients.

Advances in other local liver treatments, including, surgery and radiofrequency ablation, have also provided the opportunity for less invasive local treatment for HCC. Indeed, laparoscopic resection is a recently established method of hepatic resection that is supported by several studies ${ }^{[16,17]}$. Furthermore, robotic surgery is a promising modality in the field of surgical resection of malignant disease. With regards to liver tumor resection, due to lack of evidence, robotic surgery is not the standard of care at this time, while oncological efficacy and the perioperative outcome is under evaluation ${ }^{[18]}$. In the future, as less invasive treatment options become available, the current indication of local treatment can be overwritten. Discussion in a multi-disciplinary team, consisting of a surgeon, hepatologist, medical oncologist, and radiation oncologist, may lead to better decision making, especially in cases with adverse factors.

This study has several limitations due to the small retrospective study basis. The number of patients and events are not sufficient to perform a reliable statistical test to detect the critical adverse factors related to SBRT. Additionally, there were no highly challenging cases in the current study population; for example, poorer liver function, poorer performance status, or multiple lesions. Thus, the boundary of feasible patients on liver SBRT was not shown in this study.

In conclusion, SBRT was safely and effectively administered to a group of patients harboring both physical and tumor adverse factors as long as conducted following patient selection and dose constraints that were used in this study. Therefore, SBRT seems to be a good treatment option for patients with primary liver tumors.

\section{DECLARATIONS}

\section{Authors' contributions}

Made contributions to conception and design of the study, performed data analysis and interpretation and manuscript writing: Shimizuguchi T

Performed data acquisition, technical and material support and manuscript editing: Imamura J, Hashimoto S, Karasawa K 


\section{Availability of data and materials}

Not applicable.

\section{Financial support and sponsorship}

None.

\section{Conflicts of interest}

All authors declared that there are no conflicts of interest.

\section{Ethical approval and consent to participate}

This study was approved by the Institutional Review Board of Tokyo Metropolitan Cancer and Infectious Diseases Center, Komagome Hospital (\#2450).

\section{Consent for publication}

Not applicable.

\section{Copyright}

(c) The Author(s) 2020.

\section{REFERENCES}

1. Akinyemiju T, Abera S, Ahmed M, Alam N. Global burden of disease liver cancer collaboration. the burden of primary liver cancer and underlying etiologies from 1990 to 2015 at the global, regional, and national level: results from the global burden of disease study 2015. JAMA Oncol 2017;3:1683-91.

2. Hori M, Matsuda T, Shibata A, Katanoda K, Sobue T, et al. Cancer incidence and incidence rates in Japan in 2009: a study of 32 population-based cancer registries for the Monitoring of Cancer Incidence in Japan (MCIJ) project. Jpn J Clin Oncol 2015;45:884-91.

3. Omata M, Cheng AL, Kokudo N, Kudo M, Lee JM, et al. Asia-Pacific clinical practice guidelines on the management of hepatocellular carcinoma: a 2017 update. Hepatol Int 2017;11:317-70.

4. Heimbach JK, Kulik LM, Finn RS, Sirlin CB, Abecassis MM, et al. AASLD guidelines for the treatment of hepatocellular carcinoma. Hepatology 2018;67:358-80.

5. Murray LJ, Dawson LA. Advances in stereotactic body radiation therapy for hepatocellular carcinoma. Semin Radiat Oncol 2017;27:247-55.

6. Bujold A, Massey CA, Kim JJ, Brierley J, Cho C, et al. Sequential phase I and II trial stereotactic body radiotherapy for locally advanced hepatocellular carcinoma. J Clin Oncol 2013;31:1631-9.

7. Kang JK, Kim MS, Cho CK, Yang KM, Yoo HJ, et al. Stereotactic body radiation therapy for inoperable hepatocellular carcinoma as a local salvage treatment after incomplete transarterial chemoembolization. Cancer 2012;118:5424-31.

8. Durand-Labrunie J, Baumann AS, Ayav A, Laurent V, Boleslawski E, et al. Curative irradiation treatment of hepatocellular carcinoma: a multicenter phase 2 trial. Int J Radiat Oncol Biol Phys 2020;107:116-25.

9. Charlson ME, Pompei P, Ales KL, MacKenzie CR, Tang GJ, et al. A new method of classifying prognostic comorbidity in longitudinal studies: development and validation. J Chronic Dis 1987;40:373-83.

10. Michel R, Françoise I, Laure P, Anouchka M, Guillaume P, et al. Dose to organ at risk and dose prescription in liver SBRT. Rep Pract Oncol Radiother 2017;22:96-102.

11. Ohri N, Tomé WA, Méndez Romero A, Miften M, Ten Haken RK, et al. Local control after stereotactic body radiation therapy for liver tumors. Int J Radiat Oncol Biol Phys 2018; doi: 10.1016/j.jirobp.2017.12.288.

12. Sanuki N, Takeda A, Oku Y, Mizuno T, Aoki Y, et al. Stereotactic body radiotherapy for small hepatocellular carcinoma: a retrospective outcome analysis in 185 patients. Acta Oncol 2014;53:399-04.

13. Culleton S, Jiang H, Haddad CR, Kim J, Brierley J, et al. Outcomes following definitive stereotactic body radiotherapy for patients with Child-Pugh B or C hepatocellular carcinoma. Radiother Oncol 2014;111:412-7.

14. Rosenberg SA, Henke LE, Shaverdian N, Mittauer K, Wojcieszynski AP, et al. A multi-institutional experience of MR-guided liver stereotactic body radiation therapy. Adv Radiat Oncol 2018;4:142-9.

15. Hong TS, Wo JY, Yeap BY, Ben-Josef E, McDonnell EI, et al. Multi-institutional phase ii study of high-dose hypofractionated proton beam therapy in patients with localized, unresectable hepatocellular carcinoma and intrahepatic cholangiocarcinoma. J Clin Oncol 2016;34:460-8.

16. Cheung TT, Poon RT, Yuen WK, Chok KS, Jenkins CR, et al. Long-term survival analysis of pure laparoscopic versus open hepatectomy for hepatocellular carcinoma in patients with cirrhosis: a single-center experience. Ann Surg 2013;257:506-11.

17. Yoon YI, Kim KH, Kang SH, Kim WJ, Shin MH, et al. Pure laparoscopic versus open right hepatectomy for hepatocellular carcinoma in 
patients with cirrhosis: a propensity score matched analysis. Ann Surg 2017;265:856-63.

18. Liu R, Wakabayashi G, Kim HJ, Choi GH, Yiengpruksawan A, et al. International consensus statement on robotic hepatectomy surgery in 2018. World J Gastroenterol 2019;25:1432-44. 